\title{
NMR Transversal Relaxivity of Suspensions of Lanthanide Oxide Nanoparticles
}

\author{
Małgorzata Norek, ${ }^{\dagger}$ Giovannia A. Pereira, ${ }^{\ddagger}$ Carlos F. G. C. Geraldes, ${ }^{\ddagger}$ Antonia Denkova, ${ }^{\S}$ \\ Wuzong Zhou," and Joop A. Peters*,† \\ Biocatalysis and Organic Chemistry, Department of Biotechnology, Delft University of Technology, \\ Julianalaan 136, 2628 BL Delft, The Netherlands, NMR Center and Center of Neuroscience and Cell Biology, \\ Department of Biochemistry, University of Coimbra, P. O. Box 3126, 3001-401 Coimbra, Portugal, Physical \\ Chemistry and Molecular Thermodynamics, Delft University of Technology, 2628 BL Delft, The Netherlands, \\ and School of Chemistry, University of St. Andrews, Fife KY16 9ST, United Kingdom
}

Received: March 22, 2007; In Final Form: May 11, 2007

\begin{abstract}
Aqueous suspensions of paramagnetic lanthanide oxide nanoparticles have been studied by NMR relaxometry. The observed $R_{2}^{*}$ relaxivities are explained by the static dephasing regime (SDR) theory. The corresponding $R_{2}$ relaxivities are considerably smaller and are strongly dependent on the interval between the two refocusing pulses. The experimental data are rationalized by assuming the value of the diffusion correlation time, $\tau_{\mathrm{D}}$, to be very long in a layer with adsorbed xanthan on the particle's surface. In this layer, the refocusing pulses are fully effective and $R_{2} \approx 0$. Outside this layer, the diffusion model for weakly magnetized particles was applied. From the fit of the experimental relaxation data with this model, both the particle radii $\left(r_{\mathrm{p}}\right)$ and the radii of the spheres, within which the refocusing pulses are fully effective ( $\left.r_{\text {diff }}\right)$, were estimated. The values of $r_{\mathrm{p}}$ obtained are in agreement with those determined by dynamic light scattering. Because the value of $r_{\text {diff }}$ depends on the external magnetic field $B$ and on the magnetic moment of the lanthanide of interest $\left(\mu_{\mathrm{eff}}^{2}\right)$, the $R_{2}$ relaxivity was found to be proportional to $B$ and to $\mu_{\mathrm{eff}}{ }^{2}$.
\end{abstract}

\section{Introduction}

During the last decades, the rapid progress in biochemical research has provided detailed insight into molecular recognition processes. These developments enable the design of contrast agents (CAs) for molecular imaging ${ }^{1}$ with medical diagnostic techniques including positron emission tomography (PET), single photon emission computed tomography (SPECT), and magnetic resonance imaging (MRI). MRI has a significantly higher spatial resolution $(\mu \mathrm{m})$ than radiodiagnostic techniques $(\mathrm{mm})$, but its use as a tool for the investigation of cellular molecular events in normal and pathological processes is hampered by its low sensitivity: a relatively large local concentration of CA is required (about $10^{-5} \mathrm{M}$ ) to achieve the desired contrast enhancement. ${ }^{2,3}$ Other imaging modalities such as PET, SPECT $\left(10^{-11}-10^{-12} \mathrm{M}\right)$, and optical fluorescence imaging $\left(10^{-15}-10^{-17} \mathrm{M}\right)$ are much more adequate in this respect. ${ }^{4}$

A possible approach to overcome the problems related with the low sensitivity of MRI is to apply vectorized CAs, which would bring a high payload of paramagnetic compound to the site of interest. For lanthanide ion based contrast agents, this was realized in various ways and different materials have been proposed including: Gd-loaded apoferritin, which allows the visualization of hepatocytes when the number of Gd-complexes per cell is about $4 \times 10^{7}, 5$ perfluorocarbon nanoparticles, which contain around $94200 \mathrm{Gd}^{3+}$ ions per particle providing ex-

* To whom correspondence should be addressed. E-mail: J.A.Peters@ tudelft.nl.

Biocatalysis and Organic Chemistry, Delft University of Technology

$\doteqdot$ University of Coimbra.

$\S$ Physical Chemistry and Molecular Thermodynamics, Delft University of Technology.

\# University of St. Andrews. tremely high relaxivity per particle and which have been already successfully used in molecular imaging of angiogenesis. ${ }^{6-10}$

Alternatively, this may be achieved with superparamagnetic (SPM) particles, single domain ferromagnets possessing a very high magnetic moment (around $10^{4} \mu_{\mathrm{B}}$ ). ${ }^{11,12}$ SPM particles have a much smaller effect on the $T_{1}$ water proton relaxation time than on the $T_{2}$. Their relaxivity can be well described by the quantum mechanical outer-sphere theory. Because of their small size (20-60 $\mathrm{nm}$ in diameter), the extreme motional narrowing conditions are satisfied, which state that water diffusion between SPM particles is rapid with respect to the difference in resonance frequencies of the various sites. In this regime the $T_{2}^{*}$ relaxation time is predicted to be equal to $T_{2}$. When iron-oxide particles are compartmentalized within cells, the internal magnetization of the compartment due to their presence has to be taken into account. In this situation the motional narrowing assumption breaks down, which results in $R_{2}^{*}\left(=1 / T_{2}^{*}\right)$ to be larger than $R_{2}$. Consequently, $R_{2}^{*}$-weighted MRI images are potentially the most sensitive to the presence of cellularly compartmentalized magnetized particles. ${ }^{13-15}$

Nanozeolites present another approach. $\mathrm{Gd}^{3+}$ exchanged zeolite $\mathrm{NaY}$ nanoparticles of an average size of $80 \mathrm{~nm}$, contain about $40000 \mathrm{Gd}^{3+}$ ions per particle. The longitudinal relaxivity $r_{1}\left(r_{1}\right.$ is the relaxation rate expressed in $\mathrm{s}^{-1} \mathrm{mM}^{-1} \mathrm{Gd}$ ) is limited by the water exchange between the interior of zeolites and the bulk. ${ }^{16}$ It was observed that $r_{2}$ relaxivity is independent of the pore structure of the zeolite and that it increases with the external field strength. ${ }^{17}$ In materials like $\mathrm{Ln}-\mathrm{AV}-9$, which have $\mathrm{Ln}^{3+}$ ions incorporated in the zeolite framework, direct interaction between $\mathrm{Ln}^{3+}$ ions and water molecules is impossible. As a result, they have a very low $r_{1}$ relaxivity, but at the same time they have a very strong impact on the $T_{2}$ relaxation. ${ }^{18}$ 
In this paper we present a study on lanthanide oxide $\left(\mathrm{Ln}_{2} \mathrm{O}_{3}\right)$ nanoparticles. These particles have a very high density of $\mathrm{Ln}^{3+}$ ions, and their magnetic properties are good candidates for $R_{2}$ weighted imaging, and therefore, after coating and attachment of targeting vectors, they may have potential as CAs in molecular imaging with MRI.

\section{Experimental Section}

The lanthanide oxide nanoparticles were purchased from Aldrich and had a diameter of less than $40 \mathrm{~nm}$ as determined with XRD by the supplier.

Water proton transverse relaxation times, $T_{2}$, were measured at 20, $60 \mathrm{MHz}$ (Mini-spec PC120 and PC160, respectively, spin analyzers obtained from Bruker), $200 \mathrm{MHz}$ (on a Bruker Avance-200 console connected to a $200 \mathrm{MHz}$ cryomagnet), 300 $\mathrm{MHz}$ (Varian INOVA spectrometer), $400 \mathrm{MHz}$ (Varian VXR$400 \mathrm{~S}$ spectrometer), and $500 \mathrm{MHz}$ (Varian Unity $500 \mathrm{spec}-$ trometer) using the Carr-Purcell-Meiboom-Gill pulse sequence $(\mathrm{CPMG})$. The values of $T_{2}^{*}$ were evaluated from the linewidths. All experimental values of relaxation rates were corrected for diamagnetic contributions using a solution of 1 wt $\%$ of xanthan in water.

The $\mathrm{Ln}_{2} \mathrm{O}_{3}$ suspensions for relaxometric studies were prepared by mixing the solid particles with doubly distilled water containing $1 \mathrm{wt} \%$ of xanthan gum as a surfactant and dispersing them in an ultrasonic bath for $5 \mathrm{~min}$.

The self-diffusion coefficient of the samples was measured on the $200 \mathrm{MHz}$ spectrometer equipped with a variabletemperature high-resolution diffusion probe. A PGMSE pulse sequence was used for the determination of the diffusion constants. The temperature was maintained by water circulation in the gradient coil. The calibration of the gradients was performed on pure $\mathrm{H}_{2} \mathrm{O}$.

High-resolution transmission electron microscopy (HRTEM) was performed on a Jeol JEM-2010 electron microscope operated at $200 \mathrm{kV}$.

Dynamic light scattering (DLS) was performed with a DLS/ SLS/ALV-5000 apparatus using a $35 \mathrm{~mW}$ HeNe laser with a wavelength of $633 \mathrm{~nm}$. The intensity autocorrelation function was measured at an angle of $90^{\circ}$ and analyzed with the CONTIN method. All samples were placed in an ultrasonic bath and were centrifuged prior to the DLS measurements in order to remove dust and other contaminants.

\section{Results and Discussion}

The bulk magnetic susceptibility shifts and the $R_{1}, R_{2}$, and $R_{2}^{*}$ relaxivities of aqueous suspensions of $\mathrm{Ln}_{2} \mathrm{O}_{3}$ particles ( $\mathrm{Ln}$ $=\mathrm{Nd}, \mathrm{Gd}, \mathrm{Er}, \mathrm{Dy}, \mathrm{Yb}$ ) with an average particle size of less than $40 \mathrm{~nm}$ (as determined by XRD) were measured. Suspensions of these nanoparticles in pure water were stable for several days, but upon inserting the samples in magnets with a magnetic field strength of more than $7 \mathrm{~T}$, coagulation and precipitation of the particles occurred. In order to avoid this, $1 \mathrm{wt} \%$ xanthan gum was added to the water. TEM and HRTEM images of the $\mathrm{Dy}_{2} \mathrm{O}_{3}$ nanoparticles (see Figure 1) show that these particles, in the dry form, are fiberlike agglomerates consisting of nanosized plates with a size of 5-10 nm. The measured d-spacings from the HRTEM images indicate that these particles are cubic $\mathrm{Dy}_{2} \mathrm{O}_{3}$ with $a=1.067 \mathrm{~nm}$. Unfortunately, it was impossible to perform dynamic light scattering measurements on the suspensions used for the NMR relaxometric study because the particle density and the xanthan concentration were too high. In more dilute suspensions (without xanthan), fractions with particles sizes of about 50-100 nm were observed after
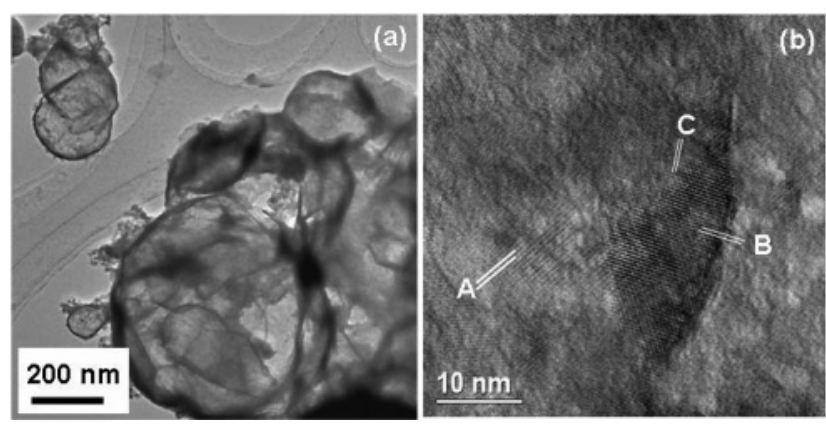

Figure 1. (a) TEM image of dysprosium oxide showing fiberlike morphology and (b) corresponding HRTEM image showing nanoparticles. The marked d-spacings are measured as (A) 0.52, (B) 0.42, and (C) $0.31 \mathrm{~nm}$, which can be indexed onto the cubic unit cell of $\mathrm{Dy}_{2} \mathrm{O}_{3}$ with $a=1.07 \mathrm{~nm}$ as (200), (-121), and (311), respectively.

TABLE 1: Comparison of $\Delta_{\mathrm{BMS}}$ of Suspensions ${ }^{a}$ of $\mathrm{Ln}_{2} \mathrm{O}_{3}$ in a Solution of $1 \mathrm{wt} \%$ of Xanthan in Water and Homogeneous Solutions Obtained after Addition of $\mathrm{HCl}$ at 7 $\mathrm{T}$ and $25^{\circ} \mathrm{C}$

\begin{tabular}{lcc}
\hline & $\Delta_{\text {BMS }} /$ ppm suspensions & $\Delta_{\text {BMS }} /$ ppm homogeneous solutions ${ }^{b}$ \\
\hline $\mathrm{Gd}$ & $0.37 \pm 0.03$ & $0.36 \pm 0.03$ \\
$\mathrm{Er}$ & $0.48 \pm 0.04$ & $0.47 \pm 0.04$ \\
$\mathrm{Dy}$ & $0.45 \pm 0.04$ & $0.49 \pm 0.05$
\end{tabular}

${ }^{a}$ Containing $1.3-1.6 \mathrm{mmol} \mathrm{Ln}_{2} \mathrm{O}_{3} / \mathrm{L}$ water. ${ }^{b}$ Obtained by adding $\mathrm{HCl}$ to suspensions; $\Delta_{\mathrm{BMS}}$ was corrected for changes in volume.

ultrasound treatment (see Figure S1 in the Supporting Information). Therefore, we assume that the agglomerates break down into a homogeneous suspension of nanoparticles upon ultrasonic treatment in the presence of xanthan.

3.1. Bulk Magnetic Susceptibility Shifts. These shifts ( $\left.\Delta_{\text {BMS }}\right)$ are related to the global magnetization $(M)$ of the lanthanide oxide particles in suspension via eq $1 .{ }^{19}$ In eq $1, B$

$$
\Delta_{B M S}=s \frac{M}{B} 10^{6}
$$

is the magnetic field strength and $s$ is a shape factor. The latter is $1 / 3$ in the present case, where the sample tube was parallel to the magnetic field during the measurement. Since magnetic coupling is negligible for $\mathrm{Ln}(\mathrm{III})$ compounds at room temperature, ${ }^{18} \mathrm{M}$ can be calculated with eq 2 . In eq $2, N$ is the number

$$
M=N n \mu_{0} \mu_{\mathrm{C}}
$$

of the particles per $\mathrm{m}^{3}, n$ is the number of $\mathrm{Ln}^{3+}$ ions per particle, $\mu_{0}$ is the vacuum magnetic permeability, and $\mu_{\mathrm{C}}$ is the Curie moment. The latter is given by eq 3 , where $\mu_{\mathrm{B}}$ is the Bohr magneton, $g$ is the Landé $g$-factor, $J$ is the quantum number of the total spin, $k$ is the Boltzmann constant, and $T$ is the absolute temperature.

$$
\mu_{\mathrm{C}}=\frac{\mu_{\mathrm{eff}}^{2} B}{3 k T}, \quad \text { where } \mu_{\mathrm{eff}}=g \mu_{\mathrm{B}} \sqrt{J(J+1)}
$$

The values of $\Delta_{\mathrm{BMS}}$ of the suspensions of nanoparticles of three lanthanides oxides were measured at $B=7 \mathrm{~T}$ and compared with $\Delta_{\mathrm{BMS}}$ of corresponding homogeneous solutions prepared directly from the suspensions concerned by adding $\mathrm{HCl}$. The data (see Table 1) show that there is no difference between the suspensions and the corresponding homogeneous solutions. It may be concluded that the global magnetizations of these systems are the same, confirming that magnetic coupling is negligible under the conditions applied. 
TABLE 2: Parameters Obtained from Analysis of $R_{2}$ and $R_{2}^{*}$ Values of Aqueous Suspensions of Lanthanide Oxides at $B=7$ T and $T=25^{\circ} \mathrm{C}^{a}$

\begin{tabular}{|c|c|c|c|c|c|c|c|}
\hline & $\Delta \omega\left(r_{\mathrm{p}}\right) 10^{6}[1 / \mathrm{s}]^{b}$ & $\Delta \omega\left(r_{\mathrm{p}}\right)_{\max } 10^{6}[1 / \mathrm{s}]^{c}$ & $\tau_{\mathrm{D}}\left(r_{\mathrm{diff}}\right) 10^{-4}[\mathrm{~s}]^{b}$ & $r_{\mathrm{p}} / r_{\text {diff }}^{b}$ & $r_{\mathrm{p}}[\mathrm{nm}]^{d}$ & $R_{2}^{0}[1 / \mathrm{s}]^{b}$ & $R_{2, \exp }^{*}\left[\mathrm{~s}^{-1}\right]^{e}$ \\
\hline $\mathrm{Nd}_{2} \mathrm{O}_{3}$ & $0.539 \pm 0.002$ & $1.928 \pm 0.002$ & $6.6 \pm 0.7$ & $0.110 \pm 0.004$ & $123 \pm 12$ & $1.7 \pm 0.1$ & $32 \pm 3$ \\
\hline $\mathrm{Gd}_{2} \mathrm{O}_{3}$ & $3.838 \pm 0.003$ & $8.525 \pm 0.003$ & $17.4 \pm 1.1$ & $0.036 \pm 0.001$ & $65 \pm 6$ & $9.9 \pm 0.2$ & $237 \pm 24$ \\
\hline $\mathrm{Dy}_{2} \mathrm{O}_{3}$ & $4.818 \pm 0.027$ & $13.776 \pm 0.027$ & $2.8 \pm 0.5$ & $0.069 \pm 0.004$ & $50 \pm 5$ & $21.8 \pm 1.6$ & $300 \pm 33$ \\
\hline $\mathrm{Er}_{2} \mathrm{O}_{3}$ & $4.180 \pm 0.006$ & $15.569 \pm 0.006$ & $5.4 \pm 0.5$ & $0.049 \pm 0.001$ & $49 \pm 5$ & $14.3 \pm 0.3$ & $238 \pm 25$ \\
\hline $\mathrm{Yb}_{2} \mathrm{O}_{3}$ & $1.333 \pm 0.004$ & $3.302 \pm 0.004$ & $4.1 \pm 0.6$ & $0.084 \pm 0.004$ & $74 \pm 7$ & $2.6 \pm 0.2$ & $68 \pm 6$ \\
\hline
\end{tabular}

${ }^{a} 1 \mathrm{mmol} \mathrm{Ln}{ }^{3+} / \mathrm{L}$ water containing $1 \mathrm{wt} \%$ xanthan. ${ }^{b}$ From fitting of experimental data with eqs 4 and $12 .{ }^{c}$ Calculated with eqs 5 and $9 .{ }^{d}$ Calculated from the best-fit values of $\tau_{\mathrm{D}}\left(r_{\mathrm{diff}}\right), r_{\mathrm{p}} / r_{\mathrm{diff}}$, and the experimentally determined value of $D_{0} .{ }^{e}$ As evaluated from the experimental linewidths.

TABLE 3: Parameters Obtained from Analysis of $R_{2}$ and $R_{2}^{*}$ Values of an Aqueous Suspension of $\mathrm{Dy}_{2} \mathrm{O}_{3}$ at $T=25{ }^{\circ} \mathrm{C}^{a}$

\begin{tabular}{|c|c|c|c|c|c|c|}
\hline$B[\mathrm{~T}]$ & $\Delta \omega\left(r_{\mathrm{p}}\right) 10^{6}[1 / \mathrm{s}]^{b}$ & $\tau_{\mathrm{D}}\left(r_{\text {diff }}\right) 10^{-4}[\mathrm{~s}]^{b}$ & $r_{\mathrm{p}} / r_{\text {diff }^{b}}^{b}$ & $r_{\mathrm{p}}[\mathrm{nm}]^{c}$ & $R_{2}^{0}[1 / \mathrm{s}]^{b}$ & $R_{2, \exp }^{*}\left[\mathrm{~s}^{-1}\right]^{\epsilon}$ \\
\hline 0.47 & $0.4^{d}$ & $35.3 \pm 2.4$ & $0.071 \pm 0.005$ & $184 \pm 18$ & $1.1 \pm 0.02$ & \\
\hline 1.4 & $1.1^{d}$ & $7.0 \pm 1.02$ & $0.077 \pm 0.005$ & $89 \pm 9$ & $3.2 \pm 0.2$ & \\
\hline 4.7 & $4.7 \pm 0.013$ & $7.3 \pm 0.99$ & $0.046 \pm 0.002$ & $54 \pm 5$ & $18.1 \pm 0.7$ & $290 \pm 80$ \\
\hline 7.0 & $4.8 \pm 0.027$ & $2.8 \pm 0.47$ & $0.069 \pm 0.004$ & $50 \pm 5$ & $21.8 \pm 1.6$ & $300 \pm 33$ \\
\hline 9.4 & $7.2 \pm 0.033$ & $9.4 \pm 1.29$ & $0.044 \pm 0.002$ & $58 \pm 6$ & $37.1 \pm 1.8$ & $454 \pm 50$ \\
\hline 11.7 & $9.6 \pm 0.097$ & $2.0 \pm 0.49$ & $0.066 \pm 0.005$ & $41 \pm 4$ & $40.9 \pm 5.6$ & $593 \pm 60$ \\
\hline
\end{tabular}

${ }^{a} 1 \mathrm{mmol} \mathrm{Dy}{ }^{3+} / \mathrm{L}$ water containing $1 \mathrm{wt} \%$ xanthan. ${ }^{b}$ From fitting of experimental data with eqs 4 and $12 .{ }^{c}$ Calculated from the best-fit values of $\tau_{\mathrm{D}}\left(r_{\mathrm{diff}}\right), r_{\mathrm{p}} / r_{\text {diff }}$, and the experimentally determined value $D_{0 .}{ }^{d}$ Extrapolated from the values of the best-fit of data measured at 4.7, 7.0, 9.4, and 11.4 T. This parameter was fixed during the fitting. ${ }^{e}$ As evaluated from the experimental linewidths.

3.2. Relaxation Rates $\left(\boldsymbol{R}_{\mathbf{1}}, \boldsymbol{R}_{\mathbf{2}}^{*}\right.$, and $\left.\boldsymbol{R}_{\mathbf{2}}\right)$. The $\mathrm{Ln}_{2} \mathrm{O}_{3}$ nanoparticles have almost no effect on longitudinal relaxation times $\left(T_{1}\right)$ of the ${ }^{1} \mathrm{H}$ water resonance; the $T_{1}$ values observed in a suspension containing $1 \mathrm{mM} \mathrm{Ln}$ were $2-3 \mathrm{~s}$, except for the suspension with $\mathrm{Gd}_{2} \mathrm{O}_{3}$, which had a $T_{1}$ value of $0.6 \mathrm{~s}$ (see Supporting Information, Table S1).

By contrast, the effects on the transversal relaxation rates were substantial. The linewidths of the ${ }^{1} \mathrm{H}$ water resonance of suspensions of the paramagnetic lanthanide oxides were large in comparison to those of the diamagnetic $\mathrm{La}_{2} \mathrm{O}_{3}$ suspension $(20 \mathrm{~Hz})$, which after subtraction of the line width for a xanthan solution in pure water gives a linewidth of $8 \mathrm{~Hz}$. From the linewidths of samples measured, values of $R_{2}^{*}$ were evaluated (see Tables 2 and 3 ).

The line-broadenings can be ascribed to susceptibility induced $R_{2}$ enhancements as a result of the diffusion of water molecules in the field inhomogeneities created by the magnetized particles. The magnetic field changes in space leading to differences in the Larmor frequencies of the protons. The proton Larmor frequency at a particular location is given by the relation $\omega=$ $\gamma B_{\mathrm{loc}}$, where $\omega$ is the proton Larmor frequency (in rad s${ }^{-1}$ ) and $B_{\text {loc }}$ is the local strength of the magnetic field. The diffusion of water protons between different magnetic environments reduces their phase coherence and, consequently, causes effective $T_{2}$ shortening.

The transverse relaxation rates $\left(R_{2}\right)$ were determined by means of the Carr-Purcell-Meiboom-Gill pulse sequence (CPMG). These measurements all showed a perfect monoexponential decay, which is characteristic for magnetic compounds enhancing the water proton relaxivity by diffusion. ${ }^{20}$ The CPMG measurements were performed as a function of the time distance between two consecutive refocusing pulses $\left(\tau_{\mathrm{CP}}\right)$ in the train of $180^{\circ}$ pulses applied..$^{21-23}$ Figures 2 and 3 show the dependence of $R_{2}$ on $\tau_{\mathrm{CP}}$ for various lanthanide oxides at a single magnetic field and for $\mathrm{Dy}_{2} \mathrm{O}_{3}$ at different magnetic fields, respectively. The values of $R_{2}$ obtained are much smaller than the corresponding $R_{2}^{*}$ values and appeared to be strongly dependent on $\tau_{\mathrm{CP}}$. This behavior is characteristic for a system in the static dephasing regime (SDR), where the condition $\tau_{\mathrm{D}}>\Delta \omega\left(r_{\mathrm{p}}\right)^{-1}$ holds $\left(\tau_{\mathrm{D}}=r_{\mathrm{p}}^{2} / D\right.$, where $r_{\mathrm{p}}$ is the radius of the particle, $D$ is the water diffusion coefficient, and $\Delta \omega\left(r_{\mathrm{p}}\right)$ is the Larmor

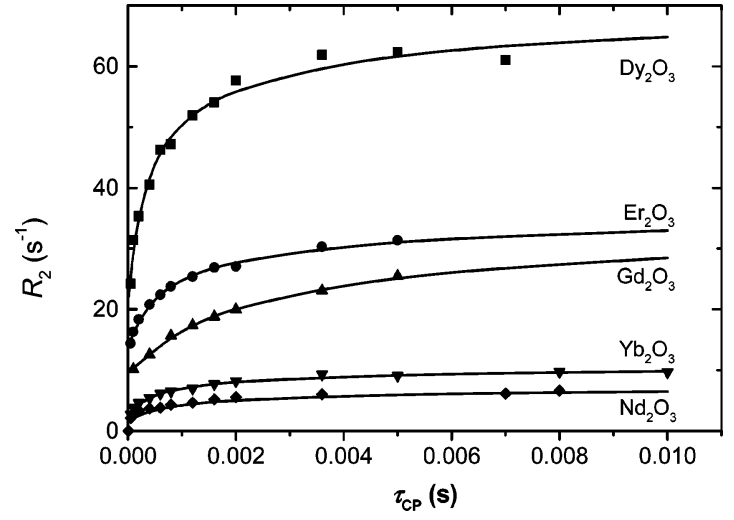

Figure 2. Dependence of $R_{2}$ on $\tau_{\mathrm{CP}}$ for different lanthanide oxide nanoparticles at $7 \mathrm{~T}$ and $25^{\circ} \mathrm{C}$; the curves are fits of the experimental data to eq 4 and 12 (for more details see text); the suspensions always contained $1 \mathrm{mmol} \mathrm{Ln}^{3+} / \mathrm{L}$ water with $1 \mathrm{wt} \%$ xanthan.

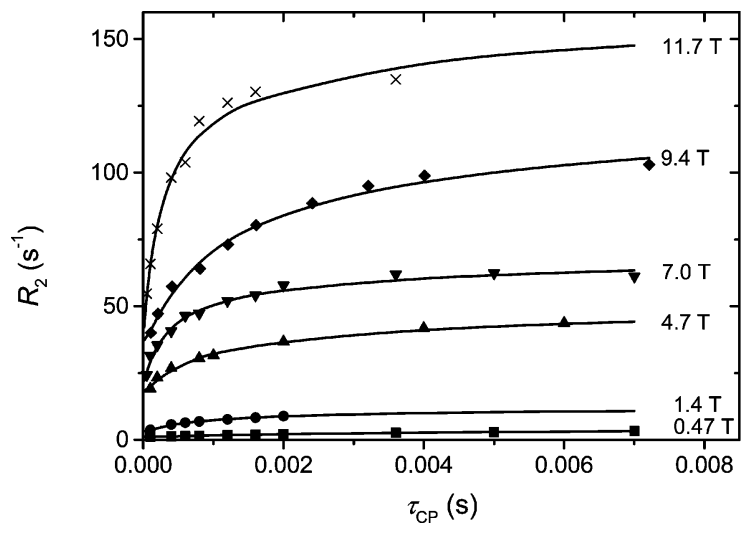

Figure 3. Dependence of $R_{2}$ on $\tau_{\mathrm{CP}}$ measured in different external magnetic fields at $25{ }^{\circ} \mathrm{C}$; the curves are fits of the experimental data to eq 4 and 12 (for more details see the text); the suspensions always contained $1 \mathrm{mmol} \mathrm{Dy}{ }^{3+} / \mathrm{L}$ water with 1 wt $\%$ xanthan.

frequency shift at the particle's surface). Under this condition, the relaxation rate $R_{2}^{*}$ can be ascribed to the dephasing of motionless magnetic moments in a nonuniform field created by randomly distributed magnetic particles. ${ }^{24-29}$ The value of $R_{2}^{*}$ is then given by eq 4 : 


$$
R_{2}^{*}=\frac{1}{T_{2}^{*}}=R_{2}^{0}+2 \pi \sqrt{3} f \Delta \omega\left(r_{\mathrm{p}}\right) / 9
$$

Here, $f=v N$ is the volume fraction occupied by the particles ( $v$ is the volume of a single particle and $N$ is the number of particles per $\mathrm{m}^{3}$ ) and $R_{2}^{0}$ is the contribution due to other relaxation mechanisms, such as the diamagnetic relaxation and a contribution as the result of chemical exchange of protons between the surface of the particles and the bulk water protons. Equation 4 was developed for spherical particles. The error introduced by this simplification may be neglected, since the magnetic field created by any particle is sensitive to its shape only in close proximity of the particle. For low concentrations of lanthanide oxide particles ( $f$ was always $\sim 10^{-5}$ ), one can assume that the majority of water protons experience field gradients created by approximately spherically shaped particulates. Since both $f$ and $\Delta \omega\left(r_{\mathrm{p}}\right)$ are not dependent on the particle radius $r_{\mathrm{p}}$, the values of $R_{2}^{*}$ are independent of the particle size as well.

The angular frequency shift at the surface of the particle, $\Delta \omega\left(r_{\mathrm{p}}\right)$, is related to the magnetization of a single particle, $M_{\mathrm{p}}$, and will be strongly dependent on the shape of the particles. Its maximal value is that of a spherical particle, for which the value of this parameter can be estimated by eq 5, where, $\gamma$ is the proton gyromagnetic ratio. ${ }^{30}$ For paramagnetic particles it

$$
\Delta \omega\left(r_{\mathrm{p}}\right)=\gamma M_{\mathrm{p}} / 3
$$

can be assumed that, upon placement in a magnetic field, a macroscopic magnetic moment is forming in each single particle, which is parallel to the external magnetic field. The magnitude of this magnetic moment is given by eqs $6-8$.

$$
\begin{gathered}
M_{p}=\frac{n}{v} \mu_{0} g \mu_{\mathrm{B}} J B_{\mathrm{J}}(x) \\
x=\frac{g J \mu_{\mathrm{B}} B}{k T} \\
B_{\mathrm{J}}(x)=\frac{2 J+1}{2 J} \operatorname{ctgh}\left(\frac{(2 J+1) x}{2 J}\right)-\frac{1}{2 J} \operatorname{ctgh}\left(\frac{x}{2 J}\right)
\end{gathered}
$$

The latter is the Brillouin function and $\mu_{0}$ and $J$ are the vacuum magnetic permeability and the quantum number of the total spin, respectively. For the temperature at which the samples were measured, $x \ll 1$, and then eq 6 can be simplified to

$$
M_{\mathrm{p}}=\frac{n}{v} \mu_{0} \mu_{\mathrm{C}}
$$

$M_{\mathrm{p}}$ was obtained using the particle volumes $(v)$ and $n$ values evaluated from their size (as determined from DLS) and the known density of the $\mathrm{Ln}_{2} \mathrm{O}_{3}$ in question. The magnitude of $\Delta \omega\left(r_{\mathrm{p}}\right)$ as determined by eqs 5 and 9 will give an upper limit of this parameter; nonspherical particles and agglomerates of particles will have lower values. Values for $\Delta \omega\left(r_{\mathrm{p}}\right)_{\max }$ as calculated from eqs 5 and 9 are included in Table 2.

The dependence of $R_{2}$ on $\tau_{\mathrm{CP}}$ for strongly magnetized particles $\left(\tau_{\mathrm{CP}} \Delta \omega\left(r_{\mathrm{p}}\right)>1\right)$ in the static dephasing regime has been analyzed by Gillis et al. and explained by the partial refocusing model. $^{29}$

The behavior of $R_{2}$ as a function of $\tau_{\mathrm{CP}}$ relies on a spatial division between an inner region $\left(\Delta \omega(r)>\tau_{\mathrm{CP}}{ }^{-1}\right)$ according to this model, where the refocusing pulses are not effective due to the extremely high gradients close to the particles surface and an outer one $\left(\Delta \omega(r)<\tau_{\mathrm{CP}}{ }^{-1}\right)$, where the refocusing pulses are partially effective due to effects of weak field inhomogeneities (far away from the particles surface). The radius of the sphere that forms the border between the two regions $\left(r_{\mathrm{SDR}}\right)$ depends on $\tau_{\mathrm{CP}}$. The overall relaxivity is considered as being the weighted sum of two components: a fast one coming from the inner and a slow one from the outer region. For relatively small $\tau_{\mathrm{CP}}$, the relaxivity is assumed to rest exclusively on the contribution from the outer region. The model predicts that upon the increase of $\tau_{\mathrm{CP}}$, the contribution from the inner region becomes increasingly important (the fast component in the signal starts to dominate), resulting in the progressive increase of the $R_{2}$ relaxivity until the maximal possible value, characterized by $R_{2}^{*}\left(R_{2}\left[\tau_{\mathrm{CP}} \rightarrow \infty\right]=R_{2}^{*}\right)$. Surprisingly, attempts to fit the present experimental data with the partial refocusing model failed (see Supporting Information); a leveling off of the curves of $R_{2}$ as a function of $\tau_{\mathrm{CP}}$ at $R_{2}^{*}$ could not be obtained, the bestfit values of $\Delta \omega(r)$ were significantly smaller than those calculated from particle sizes as determined by DLS and eq 5 and 9 , and the best-fit values of $r_{\mathrm{p}}$ were much larger than those determined by DLS, and they appeared to be strongly dependent on the magnetic field strength, which is unlikely.

We attribute this behavior to the xanthan gum that we applied as an emulsifier. It is known that it may adsorb in a thick layer on oxide surfaces. ${ }^{31}$ Therefore, the diffusion of water molecules near the surface may be relatively slow due to formation of hydrogen bonds with the adsorbed xanthan chains. If in this layer the condition $\tau_{\mathrm{D}} \gg \Delta \omega\left(r_{\mathrm{p}}\right)^{-1}$ holds, the diffusion correlation time is not effective when refocusing pulses are applied and, consequently, the phase incoherence of the water protons is fully refocused resulting in an $R_{2}$ value approaching zero. ${ }^{32}$

Let us consider this situation when no refocusing pulses are applied. Upon increase of the distance from the particle's center, $r\left(r>r_{\mathrm{p}}\right)$, protons will feel the field inhomogeneities caused by the magnetization of the particle in a decreasing extent. In the xanthan layer, we suppose that the condition $\tau_{\mathrm{D}}(r)>$ $\Delta \omega(r)^{-1}$ holds and then the effect of diffusion is negligible (SDR). Near the outside of this layer, the condition $\tau_{\mathrm{D}}(r)<$ $\Delta \omega(r)^{-1}$ will be fulfilled at some distance from the particle surface. Because, there, diffusion introduces unrecoverable losses of the phase coherence, refocusing pulses are no longer fully efficient in this part of the sample, resulting in nonzero $R_{2}$ relaxivity in the CPMG experiment. The radius of a sphere defining the boundary between the two regions $\left(r_{\text {diff }}\right)$ can be roughly estimated by assuming that this boundary is defined by $\tau_{\mathrm{D}}(r)=\Delta \omega(r)^{-1}$ (see eq 10). Then this radius is given by eq 11.

$$
\begin{aligned}
\tau_{\mathrm{D}}\left(r_{\mathrm{p}}\right)\left(\frac{r_{\text {diff }}}{r_{\mathrm{p}}}\right)^{2}\left(\frac{D}{D_{0}}\right)=\tau_{\mathrm{D}}\left(r_{\text {diff }}\right)= & \\
& \frac{1}{\Delta \omega\left(r_{\text {diff }}\right)}=\frac{1}{\Delta \omega\left(r_{\mathrm{p}}\right)}\left(\frac{r_{\text {diff }}}{r_{\mathrm{p}}}\right)^{3} \\
r_{\text {diff }}= & \frac{\Delta \omega\left(r_{\mathrm{p}}\right) r_{\mathrm{p}}{ }^{3}}{D_{0}}
\end{aligned}
$$

Here, $D_{0}$ is assumed to be unrestricted and corresponds to the majority of water protons at some distance from the surface of the particle.

For the refocusing pulses to be efficient, at the same time, the condition $\tau_{\mathrm{CP}} \ll \tau_{\mathrm{D}}$ must be fulfilled. ${ }^{33,34}$ Taking into account that the protons in the layer between the spheres with radii of $r_{\mathrm{p}}$ and $r_{\mathrm{diff}}$ do not contribute to the relaxivity, we consider the 


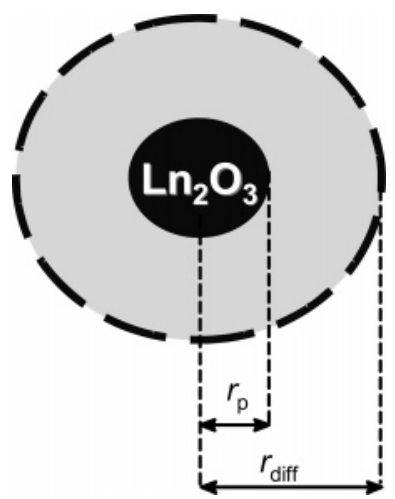

Figure 4. Schematic representation of the model applied to analyze the transverse relaxation behavior of aqueous suspensions of paramagnetic lanthanide oxides. The shaded area around the lanthanide oxide particle represents the layer, which does not contribute to the relaxivity, $R_{2}=0$.

system as consisting of magnetic particulates of radius $r_{\text {diff }}$ rather than $r_{\mathrm{p}}$ (see Figure 4). Consequently, the system is characterized by $\tau_{\mathrm{D}}\left(r_{\text {diff }}\right)$ and $\Delta \omega\left(r_{\text {diff }}\right)$. In this situation $\tau_{\mathrm{D}}\left(r_{\text {diff }}\right)$ can be relatively high, since $r_{\text {diff }}>r_{\mathrm{p}}$. The field gradient caused by the strongly magnetized particles is rapidly decreasing upon increasing the distance to the particle and, therefore, we expect that the field inhomogeneities are weak in character outside the sphere with radius $r_{\text {diff. }}$ Then, the theory of Jensen and Chandra for weak field inhomogeneities can be applied. ${ }^{35}$ Assuming a Gaussian shape of the field correlation function, these authors derived the following equations (for irregular objects and unrestricted diffusion):

$$
R_{2}=R_{2}^{0}+{ }^{1} /{ }_{2} \Delta \omega\left(r_{\text {diff }}\right)^{2} f\left(r_{\text {diff }}\right) \tau_{\mathrm{D}}\left(r_{\text {diff }}\right) F(x)
$$

where

$$
F(x)=\frac{1}{\sqrt{\pi}} \int_{0}^{\infty} \mathrm{d} y \frac{\mathrm{e}^{-y}}{\sqrt{y}}\left[1-\frac{1}{x y} \tanh (x y)\right] \text { and } x=\frac{4 \tau_{\mathrm{CP}}}{\tau_{\mathrm{D}}\left(r_{\mathrm{diff}}\right)}
$$

The values of $\Delta \omega\left(r_{\text {diff }}\right), \tau_{\mathrm{D}}\left(r_{\mathrm{diff}}\right)$, and $f\left(r_{\mathrm{diff}}\right)$ can be expressed as follows:

$$
\begin{gathered}
\Delta \omega\left(r_{\text {diff }}\right)=\Delta \omega\left(r_{\mathrm{p}}\right)\left(\frac{r_{\mathrm{p}}}{r_{\text {diff }}}\right)^{3} \\
\tau_{\mathrm{D}}\left(r_{\text {diff }}\right)=\tau_{\mathrm{D}}\left(r_{\mathrm{p}}\right)\left(\frac{r_{\text {diff }}}{r_{\mathrm{p}}}\right)^{2}\left(\frac{D}{D_{0}}\right) \\
f\left(r_{\text {diff }}\right)=f\left(r_{\mathrm{p}}\right)\left(\frac{r_{\text {diff }}}{r_{\mathrm{p}}}\right)^{3}
\end{gathered}
$$

For $x \ll 1, F(x)=x^{2} / 4$, and for $x \gg 1, F(x)=1$. Thus,

$$
\begin{aligned}
R_{2}=R_{2}^{0}+\frac{1}{2} \Delta \omega\left(r_{\text {diff }}\right)^{2} f\left(r_{\text {diff }}\right) \frac{\tau_{\mathrm{CP}}^{2}}{\tau_{\mathrm{D}}\left(r_{\text {diff }}\right)} \\
\text { for } \tau_{\mathrm{CP}} \ll \tau_{\mathrm{D}}\left(r_{\text {diff }}\right)
\end{aligned}
$$

and

$$
\begin{array}{r}
R_{2}=R_{2}^{0}+\frac{1}{2} \Delta \omega\left(r_{\text {diff }}\right)^{2} f\left(r_{\text {diff }}\right) \tau_{\mathrm{D}}\left(r_{\text {diff }}\right) \\
\\
\text { for } \tau_{\mathrm{CP}} \gg \tau_{\mathrm{D}}\left(r_{\text {diff }}\right)
\end{array}
$$
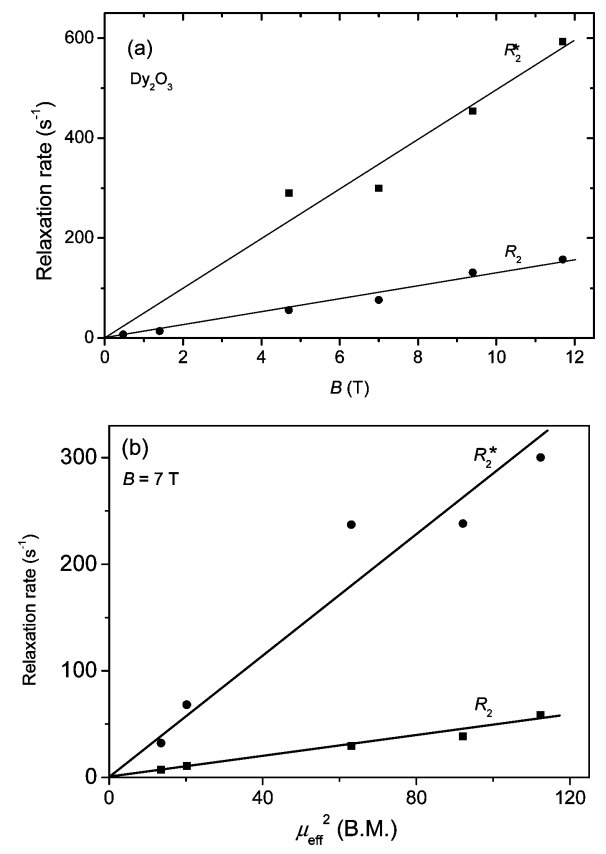

Figure 5. (a) The dependence of $R_{2}$ (slope $13.0 \mathrm{~s}^{-1} \mathrm{~T}^{-1} ; r=0.992$ ) and $R_{2}^{*}$ (slope $49.5 \mathrm{~s}^{-1} \mathrm{~T}^{-1} ; r=0.985$ ) of an aqueous suspension of $\mathrm{Dy}_{2} \mathrm{O}_{3}$ on the external magnetic field $B$. (b) The dependence of $R_{2}$ (slope $0.48 \mathrm{~s}^{-1} \mu_{\mathrm{B}}^{-2} ; r=0.989$ ) and $R_{2}^{*}$ (slope $2.8 \mathrm{~s}^{-1} \mu_{\mathrm{B}}^{-2} ; r=0.972$ ) of an aqueous suspension of lanthanide oxide nanoparticles on $\mu_{\text {eff }}^{2}$. All $R_{2}$ values presented in the graphs were calculated for $\tau_{\mathrm{CP}}=1 \mathrm{~s}$, and the concentrations of the samples were about $0.5 \mathrm{mM}$.

The experimental data $\left(R_{2}\right.$ and $\left.R_{2}^{*}\right)$ were fitted simultaneously with eqs 4 and 12 using $\Delta \omega\left(r_{\mathrm{p}}\right), \tau_{\mathrm{D}}\left(r_{\text {diff }}\right), r_{\mathrm{p}} / r_{\text {diff }}$, and $R_{2}^{0}$ as adjustable parameters. An excellent agreement between the experimental and calculated values was achieved. The bestfit parameters are listed in Tables 2 and 3, whereas the calculated curves are displayed in Figures 2 and 3 . The values $\tau_{\mathrm{D}}\left(r_{\text {diff }}\right)$ are all in the milliseconds range, which is in agreement with the observed dependence of $R_{2}$ on $\tau_{\mathrm{CP}}$. The smallest $\tau_{\mathrm{CP}}$ value applied was $5 \times 10^{-5} \mathrm{~s}$, so it is evident that the condition $\tau_{\mathrm{CP}}$ $\ll \tau_{\mathrm{D}}\left(r_{\text {diff }}\right)$ is met. When $\tau_{\mathrm{CP}}$ approaches $\tau_{\mathrm{D}}\left(r_{\text {diff }}\right)$, the system reaches the motional narrowing regime, where refocusing pulses are no longer efficient and, consequently, the curves of $R_{2}$ versus $\tau_{\mathrm{CP}}$ levels off at $\tau_{\mathrm{CP}}>\tau_{\mathrm{D}}\left(r_{\text {diff }}\right)$. The obtained values of $\tau_{\mathrm{D}}\left(r_{\text {diff }}\right)$ (see Tables 2 and 3 ) correspond very well with the values of $\tau_{\mathrm{CP}}$, where the curves start to saturate (see Figures 2 and 3 ). For example, the longest $\tau_{\mathrm{D}}\left(r_{\text {diff }}\right)$ was obtained for $\mathrm{Gd}_{2} \mathrm{O}_{3}$, and this is reflected in a relatively low initial slope of the corresponding curve.

$r_{\mathrm{p}}$ can be determined by knowing $\tau_{\mathrm{D}}\left(r_{\mathrm{diff}}\right), r_{\mathrm{p}} / r_{\text {diff }}$, and the diffusion coefficient $D_{0}$. $D_{0}$ was measured by the pulsed gradient multiple spin echo pulse sequence, PGMSE, and was found to be $1.9 \times 10^{-9} \mathrm{~m}^{2} \mathrm{~s}^{-1}$. The resulting values of $r_{\mathrm{p}}$, gathered in Tables 2 and 3, are in good agreement with the results of the DLS analyses. The evaluated particle radii show a decreasing trend upon increase of the magnetic field (see Table 3), which probably is due to the crudeness of the model applied.

The best-fit values of $R_{2}^{0}$ are considerable (see Tables 2 and 3 ). The diamagnetic contribution to $R_{2}^{0}$ was estimated from measurements on suspensions of $\mathrm{La}_{2} \mathrm{O}_{3}$ nanoparticles and appeared to be less than 1 . Possibly $R_{2}^{0}$ includes an additional contribution from the chemical exchange between protons on the particles surface and bulk water. However, $R_{2}^{0}$ depends linearly on both $B$ and $\mu_{\text {eff }}^{2}$ while for chemical exchange a quadratic dependence would be expected. Therefore, it cannot 
be excluded that the values $R_{2}^{0}$ also include a contribution that corrects for the simplifications in the model.

The values of $R_{2}^{*}$ and the saturation values of $R_{2}$ are both proportional to the external magnetic field $B$ as well as to $\mu_{\text {eff }}^{2}$ (see Figure 5). For $R_{2}^{*}$, this is in agreement with eq 4 , which shows a linear relationship between $R_{2}^{*}$ and $\Delta \omega\left(r_{\mathrm{p}}\right)$, which is proportional to $B$ and $\mu_{\mathrm{eff}}{ }^{2}$.

Substitution of eqs 13-15, which relate $\Delta \omega\left(r_{\text {diff }}\right), \tau_{\mathrm{D}}\left(r_{\text {diff }}\right)$, and $f\left(r_{\text {diff }}\right)$ to $\Delta \omega\left(r_{\mathrm{p}}\right), \tau_{\mathrm{D}}\left(r_{\mathrm{p}}\right)$, and $f\left(r_{\mathrm{p}}\right)$, respectively, for spherical particles, in eq 17 , gives eq 18 . Taking into consideration eq

$$
R_{2}=R_{2}^{0}+\frac{1}{2} \Delta \omega\left(r_{\mathrm{p}}\right)^{2} f\left(r_{\mathrm{p}}\right) \tau_{\mathrm{D}}\left(r_{\mathrm{p}}\right)\left(\frac{r_{\mathrm{p}}}{r_{\text {diff }}}\right)\left(\frac{D}{D_{0}}\right)
$$

11 , which gives a rough estimate of $r_{\text {diff, }}$, it can be concluded that $R_{2} \approx \Delta \omega\left(r_{\mathrm{p}}\right) f$. Since $\Delta \omega\left(r_{\mathrm{p}}\right)$ is proportional to $B$ and $\mu_{\mathrm{eff}}{ }^{2}$, and the results of the fittings described above show that the same holds for $R_{2}^{0}$, it can be concluded that $R_{2}$ is proportional to $B$ and $\mu_{\mathrm{eff}}{ }^{2}$ as well, which is in agreement with the results presented in Figure 5.

Equation 18 also demonstrates that $R_{2}$ is proportional to the diffusion constant in close proximity of the particle's surface $(D)$ relative to that of bulk water $\left(D_{0}\right)$. This is in agreement with the expected increase of the importance of the relaxation process due to diffusion of water protons in local magnetic field gradients upon increase of $D$ and thus with increasing distance to the surface of the particle, since more efficient diffusion leads to less recovery of phase coherence by the refocusing pulses. ${ }^{21}$

\section{Conclusions}

Paramagnetic $\mathrm{Ln}_{2} \mathrm{O}_{3}$ particles behave as strongly magnetized particles and, as a result of the magnetic field inhomogeneities, they induce large ${ }^{1} \mathrm{H}$ transverse relaxation rate enhancements in aqueous suspensions. Although these particles show paramagnetic behavior, ${ }^{36-39}$ the magnitude of this effect is comparable with the effect caused by SPM particles. ${ }^{40-43}$ For instance, AMI25 dextran coated magnetite particles have $M_{\mathrm{p}}=8.2 \times$ $10^{-2} \mathrm{~T},{ }^{25}$ while for $\mathrm{Dy}_{2} \mathrm{O}_{3} M_{\mathrm{p}}$ can be calculated to be $16.9 \times$ $10^{-2} \mathrm{~T}$ at $B=7 \mathrm{~T}$. However, the field dependence of the global magnetizations of the particle suspensions differs significantly. At a weak external magnetic field $(B<1 \mathrm{~T})$, the values of $M$ for SPMs are about 5 orders of magnitude larger than those for paramagnetic particles and get saturated already in a magnetic field of about $1 \mathrm{~T}$.

Both $R_{2}^{*}$ and $R_{2}$ values of $\mathrm{Ln}_{2} \mathrm{O}_{3}$ nanoparticles are linearly dependent on the strength of the external magnetic field, $B$. At present, there is a tendency to perform MRI exams at higher magnetic fields. Lanthanide oxides have favorable relaxivity properties for these higher fields.

The results described suggest that an $R_{2}$-silent layer exists around the particles, which may be attributed to adsorption of xanthan, which was applied as an emulsifier. It may be expected that much higher $R_{2}$ relaxivities will be obtained when the particles are surface treated with a thinner layer of coating. Optimally, paramagnetic lanthanide oxide nanoparticles will behave as strongly magnitized particles, and then the highest relaxivities should be expected for particle radii which are such that the system is on the border between the regions where the outer sphere and the SDR theories are valid. ${ }^{29}$

For the practical application as a MRI contrast agent, the lanthanide oxide particles should be protected against leaching of highly toxic free $\mathrm{Ln}^{3+}$ ions by an appropriate coating, for example, with a dextran of a polysiloxane shell. ${ }^{44}$ Attachment of additional functions, such as PEG groups, may improve the biodistribution and, furthermore, these particles can, if conjugated to the appropriate targeting vector, deliver a high payload of $\mathrm{Ln}^{3+}$ at the site of interest. The results of the present study may be helpful for the design of particles with optimal size and thickness of surface coatings.

Recently, McDonald and Watkin have shown that $\mathrm{Gd}_{2} \mathrm{O}_{3}$ nanoparticles become superparamagnetic upon coating. ${ }^{45,46} \mathrm{It}$ may be expected that the oxides of other paramagnetic lanthanides will also become superparamagnetic upon coating. Further studies to investigate this are in progress.

Acknowledgment. We thank Prof. R. N. Muller, Prof. P. Gillis, and Dr. A. Roch of the University of Mons-Hainaut, Belgium, for their kind help in measuring relaxivities at low magnetic fields and for helpful discussions. Thanks are due to the EU for financial support via a Marie Curie training site host fellowship (Grant MEST-CT-2004-7442), to the Foundation of Science and Technology (FCT), Portugal, (Project POCTI/QUI/ 47005/2002), and to FEDER. This work was done in the frame of COST Action D38 "Metal-Based Systems for Molecular Imaging Applications" and the EU Network of Excellence European Molecular Imaging Laboratory" (EMIL, Grant LSCH2004-503569).

Supporting Information Available: DLS pictures of lanthanide oxide particles, $T_{1}$ values of ${ }^{1} \mathrm{H}$ water resonances in suspensions of lanthanide oxides, and fitting of the experimental $R_{2}$ data using the partial refocusing model. This material is available free of charge via the Internet at http://pubs.acs.org.

\section{References and Notes}

(1) Weissleder, R.; Mahmood, U. Radiology 2001, 219, 316-333.

(2) Ahrens, E. T.; Rothbächer, U.; Jacobs, R. E.; Fraser, S. E. Proc. Natl. Acad. Sci. U.S.A. 1998, 95, 8443-8448. 447.

(3) Mills, P. H.; Ahrens, E. T. Magn. Reson. Med. 2007, 57, 442-

(4) Weissleder, R. Radiology 1999, 212, 609-614.

(5) Aime, S.; Cabella, C.; Colombatto, S.; Crich, S. G.; Gianolio, E.; Maggioni, F. J. Magn. Reson. Imaging 2002, 16, 394-406.

(6) Morawski, A. M.; Winter, P. M.; Crowder, K. C.; Caruthers, S. D.; Fuhrhop, R. W.; Scott, R. W.; Robertson, J. D.; Abendschein, D. R. Lanza, G. M.; Wickline, S. A. Magn. Reson. Med. 2004, 51, 480-486.

(7) Winter, P.; Athey, P.; Kiefer, G.; Gulyas, G.; Frank, K.; Fuhrhop, R.; Robertson, D.; Wickline, S. A.; Lanza, G. J Magn. Magn. Mater. 2005 293, 540-545.

(8) Winter, P. M.; Caruthers, S. D.; Kassner, A.; Harris, T. D.; Chinen, L. K.; Allen, J. S.; Lacy, E. K.; Zhang, H.; Robertson, J. D.; Wickline, S. A.; Lanza, G. M. Cancer Res. 2003, 63, 5838-5843.

(9) Winter, P. M.; Morawski, A. M.; Caruthers, S. D.; Fuhrhop, R. W.; Zhang, H.; Wiliams, T. A.; Allen, J. S.; Lacy, E. K.; Robertson, J. D.; Lanza, G. M.; Wickline, S. A. Circulation 2003, 108, 2270-2274.

(10) Flacke, S.; Fisher, S.; Scott, M. J.; Fuhrhop, R.; Allen, J. S.; McLean, M.; Winter, P.; Sicard, G. A.; Gaffney, P. J.; Wickline, S. A.; Lanza, G. M. Circulation 2001, 104, 1280-1285.

(11) Muller, R. N.; Roch, A.; Colet, J.-M.; Ouakssim, A.; Gillis, P. In The Chemistry of Contrast Agents in Medical Magnetic Resonance Imaging; Merbach, A. É.; Tóth, É., Eds.; John Wiley and Sons: Chichester, U.K., 2001; Chapter 10.

(12) Muller, R. N.; Vander Elst, L.; Roch, A.; Peters, J. A.; Csajbók,

É.; Gillis. P.; Gossuin, Y. Adv. Inorg. Chem. 2005, 57, 239-292.

(13) Bowen, C. V.; Zhang, X.; Saab, G.; Gareau, P. J.; Rutt, B. K. Magn. Reson. Med. 2002, 48, 52-61.

(14) Foster-Gareau, P.; Heyn, C.; Alejski, A.; Rutt, B. K. Magn. Reson. Med. 2003, 49, 968-971.

(15) Heyn, C.; Bowen, C. V.; Rutt, B. K.; Foster, P. J. Magn. Reson. Med. 2005, 53, 312-320.

(16) Platas-Iglesias, C.; Vander Elst, L.; Zhou, W.; Muller, R. N.; Geraldes, C. F. G. C.; Maschmeyer, T.; Peters, J. A. Chem.-Eur. J. 2002, 8,22 .

(17) Csajbók, É.; Bányai, I.; Vander Elst, L.; Muller, R. N.; Zhou, W.; Peters, J. A. Chem.-Eur. J. 2005, 11, 4799-4807.

(18) Pereira, G. A.; Ananias, D.; Rocha, J.; Amaral, V. S.; Muller, R. N.; Vander Elst, L.; Tóth, É.; Peters, J. A.; Geraldes, C. F. G. C. J. Mater Chem. 2005, 15, 3832-3837. 
(19) Chu, S. C.-K.; Xu, Y.; Balschi, J. A.; Springer, C. S., Jr. Magn. Reson. Med. 1990, 13, 239-262.

(20) Gossuin Y.; Roch A.; Muller R. N.; Gillis, P. J. Magn. Reson. 2002, $158,36-42$

(21) Hardy, P. A.; Henkelman, R. M. Magn. Reson. Imaging 1989, 7, $265-275$.

(22) Hardy, P. A.; Henkelman, R. M. Magn. Reson. Med. 1991, 17, $348-356$.

(23) Jensen, J. H.; Chandra, R. Mag. Reson. Med. 2000, 43, 226-236.

(24) Brown, R. J. S. Phys. Rev. 1961, 121, 1379-1382.

(25) Muller, R. N.; Gillis, P.; Moiny, F.; Roch, A. Magn. Reson. Med. 1991, 22, 178-182.

(26) Weisskoff, R. M.; Zuo, C. S.; Boxerman, J. L.; Rosen, B. R. Magn Reson. Med. 1994, 31, 601-610.

(27) Yablonskiy, D. A.; Haacke, E. M. Magn. Reson. Med. 1994, 32, 749-763.

(28) Koenig S. H. Invest. Radiol. 1998, 11, 822-827.

(29) Gillis, P.; Moiny, F.; Brooks, R. A. Magn. Reson. Med. 2002, 47, 257-263.

(30) Roch, A.; Gossuin, Y.; Muller, R. N.; Gillis, P. J. Magn. Magn. Mater. 2005, 293, 532-539.

(31) Li, H.-Y. Chem. Res. Chin. Univ. 2004, 20, 501-503.

(32) Yung K.-T. Magn. Reson. Imaging 2003, 21, 451-463.

(33) Brooks, R. A.; Moiny, F.; Gillis, P. Magn. Reson. Med. 2001, 45, $1014-1020$

(34) Gossuin, Y.; Roch, A.; Muller, R. N.; Gillis, P. Magn. Reson. Med. 2000, 43, 237-243. 156.

(35) Jensen, J. H.; Chandra, R. Magn. Reson. Med. 2000, 44, 144-

(36) Nelson, J. A.; Bennett, L. H.; Wagner, M. J. J. Am. Chem. Soc. 2002, 124, 2979.

(37) Nelson, J. A.; Bennett, L. H.; Wagner, M. J. J. Mater. Chem. 2003, $13,857-860$. 80 .

38) Rowley, A. T.; Parkin, I. R. Inorg. Chim. Acta 1993, 211, 77-

(39) Roberts, D.; Zhu, W. L.; Frommen, C. M.; Rosenzweig, Z. J. Appl. Phys. 2000, 87, 6208-6210.

(40) Pankhurst, Q. A.; Connolly, J.; Jones, S. K.; Dobson, J. J. Phys. D: Appl. Phys. 2003, 36, R167-R181.

(41) Bulte, J. W. M.; Brooks, R. A.; Moskowitz, B. M.; Bryant, L. H., Jr.; Frank, J. A. Magn. Reson. Med. 1999, 42, 379-384.

(42) Brooks, R. A.; Vymazal, J.; Goldfarb, R. B.; Bulte, J. W. M.; Aisen, P. Magn. Reson. Med. 1998, 40, 227-235.

(43) Mornet, S.; Vasseur, S.; Grasset, F.; Dugnet, E. J. Mater. Chem. 2004, 14, 2161-2175.

(44) Bridot, J.-L.; Faure, A.-C.; Laurent, S.; Rivière, C.; Billotey, C.; Hiba, B.; Janier, M.; Josserand, V.; Coll, J.-L.; Vander Elst, L.; Muller, R.; Roux, S.; Perriat, P.; Tillement, O. J. Am. Chem. Soc. 2007, 129, 50765084.

(45) McDonald, M. A.; Watkin, K. L. Invest. Radiol. 2003, 38, 305310.

(46) McDonald, M. A.; Watkin, K. L. Acad. Radiol. 2006, 13, 421427. 